\section{Fatores associados à morbidade respiratória entre 12 e 36 meses de vida de crianças nascidas de muito baixo peso oriundas de uma UTI neonatal pública}

\author{
Risk factors for respiratory morbidity at 12 to 36 \\ months in very low birth weight premature \\ infants previously admitted to a public \\ neonatal intensive care unit
}

Geórgia Chalfun ${ }^{1}$

Rosane Reis de Mello 1

Maria Virginia Peixoto Dutra 1

Valeska Lima Andreozzi 2

Kátia Silveira da Silva 1

\footnotetext{
1 Instituto Fernandes Figueira, Fundação Oswaldo Cruz, Rio de Janeiro, Brasil. 2 Centro de Estatística e Aplicações, Universidade de Lisboa, Lisboa, Portugal.

Correspondência R. R. Mello

Departamento de Neonatologia, Instituto Fernandes Figueira Fundação Oswaldo Cruz. Rua Eurico Cruz 23, apto. 301, Rio de Janeiro, $R J$ 22461-200, Brasil. rosanemello@gmail.com rmello@iff.fiocruz.br
}

\begin{abstract}
The aim of this paper was to estimate respiratory morbidity and its determinants for premature infants aged 12 to 36 months. The population comprised 84 infants from a cohort of very low birth weight premature infants. The outcome was the respiratory morbidity incidence rate. The relationship between the independent variables and respiratory morbidity was estimated using a Poisson regression model. From 12 to 24 months of age, 56.3\% of children had experienced at least one episode of respiratory disease. $>$ From 24 to 36 months, $38.1 \%$ of children were affected. Variables significantly associated with respiratory morbidity were bronchopulmonary dysplasia $(R R=$ 1.9; 95\%CI: 1.2-2.9), abnormal lung compliance $(R R=1.6$; 95\%CI: 1.1-2.3), neonatal pneumonia $(R R=2.8 ; 95 \% C I: 1.9-4.0)$, patent ductus arteriosus ( $R R=1.6$; 95\%CI: 1.1-2.5), and respiratory morbidity in the first year of life $(R R=1.7 ; 95 \% C I$ : 1.2-2.5). The incidence of respiratory morbidity remains high in this group of high-risk infants, which calls for regular follow-up and effective interventions to prevent respiratory disease and to improve the quality of life of these children and their families.
\end{abstract}

Premature Infant; Low Birth Weight Infant; Respiratory Tract Diseases

\section{Introdução}

Sintomas respiratórios acometem cerca de $30 \%$ das crianças nascidas prematuras em idades entre 2 e 4 anos 1 . Esses recém-nascidos necessitam de suporte ventilatório por períodos prolongados, dependem do uso de oxigenoterapia e freqüentemente desenvolvem displasia broncopulmonar. A necessidade de internações hospitalares e a ocorrência de doença do trato respiratório são mais freqüentes nos primeiros anos de vida deste grupo ${ }^{2}$, e as crianças previamente acometidas por displasia broncopulmonar têm chance duas vezes maior de necessitar de internação por problemas respiratórios do que os sem displasia broncopulmonar 3,4 . As crianças que nascem prematuras apresentam com maior freqüência tosse crônica e sibilos na infância e na adolescência 5 . Além do baixo peso ao nascer e condições de saúde ao nascimento, outros fatores como sexo masculino, baixa renda familiar, exposição ao fumo, desmame precoce, menor escolaridade materna e maior densidade domiciliar são considerados fatores de risco para hospitalização em menores de cinco anos 6 .

Fatores como: uso de ventilação mecânica, pneumonia neonatal e alteração na função pulmonar apresentaram importante repercussão na incidência de morbidade respiratória no primeiro ano de vida de crianças nascidas prematuras 7,8,9, porém no nosso meio ainda é pouco conhecido se a morbidade respiratória nessas crianças se 
mantém elevada e se tal associação se mantém relevante em relação à morbidade respiratória na infância, entre 1 e 3 anos de vida.

O objetivo deste estudo foi estimar a morbidade respiratória em prematuros de muito baixo peso entre 12 e 36 meses de vida e verificar a associação da morbidade respiratória com fatores neonatais e pós-neonatais.

\section{Materiais e métodos}

\section{Delineamento e população de estudo}

Uma coorte de 97 crianças prematuras admitidas na UTI neonatal do Instituto Fernandes Figueira, Fundação Oswaldo Cruz (IFF/FIOCRUZ) no período entre janeiro de 1998 e agosto de 2000 foi acompanhada no ambulatório de seguimento de recém-nascidos de risco desde a alta da unidade neonatal, quando se iniciou a coleta de dados.

Os critérios de inclusão adotados foram: peso de nascimento menor do que $1.500 \mathrm{~g}$ e idade gestacional inferior a 34 semanas, adequadas para a idade gestacional. A idade gestacional foi estimada pela data da última menstruação, ou na incerteza da data, pela ultra-sonografia obstétrica realizada no primeiro trimestre de gestação. $\mathrm{Na}$ ausência destas duas informações, foi considerada a idade gestacional obtida pelo escore New Ballard, que consiste na avaliação das características somáticas e avaliação neurológica do recém-nascido. Foram excluídas as crianças com malformações, infecções congênitas, síndromes genéticas, os pequenos para a idade gestacional e os óbitos ocorridos antes da alta da unidade neonatal.

A rotina de acompanhamento das crianças durante o primeiro ano de vida foi mensal 8,9, as informações referentes ao período (variáveis sócio-demográficas, maternas, neonatais e pós-neonatais) foram publicadas previamente 7,8,9 enquanto o acompanhamento, a partir de 12 meses de vida, se caracterizou por consultas trimestrais. A cada consulta trimestral realizada eram coletadas as fichas com registros dos responsáveis sobre a presença de intercorrências respiratórias ocorridas desde a última consulta até a consulta atual, portanto, se a consulta ocorreu no trimestre imediatamente anterior, o registro seria referente a um período de observação de três meses. Caso o intervalo entre as consultas fosse de dois trimestres, seria considerado um tempo de acompanhamento de seis meses e assim sucessivamente até o oitavo trimestre quando foi considerado um período de observação de 24 meses.

Para aumentar a adesão das famílias ao programa de seguimento desses bebês de alto risco, algumas estratégias foram empreendidas: no primeiro contato da pediatra (responsável pela pesquisa) com as famílias, antes da alta hospitalar e na primeira consulta após a alta, eram explicadas as principais intercorrências clínicas que podem afetar as crianças nascidas prematuras, explicados os objetivos da pesquisa e a importância do acompanhamento regular.

Outra estratégia adotada foi a marcação, no dia da primeira consulta no ambulatório, de todas as consultas mensais durante o primeiro ano de vida de modo a facilitar a programação ao comparecimento das famílias. No final do primeiro ano eram agendadas as consultas para o segundo ano e, no final deste, para o terceiro ano de vida em agendamentos trimestrais. No caso de ocorrerem faltas às consultas agendadas, a pediatra responsável pela pesquisa entrava em contato telefônico com o responsável pela criança, perguntava sobre o motivo do não-comparecimento e reforçava a importância da presença às consultas; então novo agendamento era feito. No caso de três faltas consecutivas às consultas, a assistente social entrava em contato com as famílias solicitando o comparecimento ao hospital.

\section{Tamanho amostral}

Considerando incidência de $25 \%$ de doença respiratória no grupo de prematuros não expostos, risco relativo de 2,5, nível de significância de 95\% e poder de $80 \%$, foi estimado um tamanho amostral de 73 crianças.

\section{Desfecho de interesse e covariáveis}

O desfecho do estudo foi a taxa de incidência de morbidade respiratória após 12 meses até 36 meses de idade. A morbidade respiratória foi definida como a presença de sibilos recorrentes e/ou pneumonia e/ou internações hospitalares por causa respiratória. O numerador desta taxa foi o total de episódios de morbidade respiratória ocorridos no período. O denominador foi composto pelo total de crianças-ano observadas. $\mathrm{O}$ tempo de acompanhamento foi calculado pelo somatório de trimestres compreendidos entre o primeiro e o último comparecimento à consulta, na faixa etária entre 12 e 36 meses, convertidos para unidade de tempo (criança/ano).

Covariáveis sócio-demográficas, tabagismo dos pais, amamentação e história de alergia nos pais e/ou irmãos foram investigadas para identificação de potenciais fatores de confusão na relação das características neonatais e pós-neonatais com o desfecho. Características neonatais como idade gestacional (analisada na forma contínua e categórica como maior e menor que 28 sema- 
nas), displasia broncopulmonar, persistência do canal arterial, uso de ventilação mecânica, pneumonia neonatal, complacência e resistência pulmonares e pós-neonatais (ter apresentado morbidade respiratória no primeiro ano de vida) foram também avaliados.

Considerou-se displasia broncopulmonar a utilização de oxigenoterapia por um período superior a 28 dias e classificou-se como displasia broncopulmonar moderada/grave a utilização de oxigenoterapia às 36 semanas de idade corrigida 10 .

Definiu-se sibilos recorrentes como a presença de dois ou mais episódios de sibilos que causassem falta de ar, cansaço ou dificuldade respiratória 11 constatados mediante ausculta pulmonar realizada por pediatra, necessitando do uso de medicação broncodilatadora e/ou aumento do tempo expiratório à ausculta pulmonar e/ou hiperinsuflação na radiografia de tórax ${ }^{12}$. Internação por problemas respiratórios referiu-se à permanência da criança em ambiente hospitalar por um período superior a 24 horas 13 .

O diagnóstico de pneumonia foi considerado nos casos de dificuldade respiratória, taquipnéia, retração inter e subcostal; estertoração crepitante e comprovada alteração radiológica (consolidação) 13,14 .

Foram realizadas provas de função pulmonar próximo à alta dos bebês da unidade neonatal. Os exames foram realizados por um médico que desconhecia a história clínica dos bebês. Os exames foram realizados com o bebê dentro de uma incubadora e durante o sono não REM, sem sedação 7. A avaliação da função pulmonar constou das medidas dos seguintes sinais fisiológicos: fluxo, pressão esofagiana e pressão de vias aéreas. Para a medida do fluxo de ar nas vias aéreas utilizou-se um pneumotacógrafo (Fleisch 00; Hans Rudolph Inc., Kansas City, Estados Unidos) e um transdutor de pressão ultrasensível (Validyne MP45; Validyne Corp., Northridge, Califórnia, Estados Unidos). A pressão esofagiana foi medida por meio de um cateter preenchido por água posicionado na altura do terço distal do esôfago. Para a pressão de vias aéreas foi empregado um transdutor de pressão Validyne DP 45. O volume corrente foi obtido pela integração eletrônica do sinal de fluxo coletado. Foram selecionados entre 20 e 50 ciclos respiratórios por arquivo gravado para o cálculo da resistência e complacência pulmonares pela utilização da técnica de regressão linear (cálculo computadorizado Abreath-Anadat/Labdat; Meakies-Christies, Montreal, Canada). Cada arquivo colhido durou vinte segundos. Os ciclos respiratórios selecionados foram os que demonstraram coeficiente de determinação igual ou superior a 0,95 (cálculo computadorizado Abreath-Anadat/Labdat).

Os valores da complacência pulmonar foram calculados e corrigidos pelo peso corpóreo.

Os resultados da complacência e resistência pulmonares foram dicotomizados, sendo considerada complacência anormal quando menor que $1,2 \mathrm{~mL} / \mathrm{cmH}_{2} \mathrm{O} / \mathrm{kg}$ ) e resistência anormal quando superior a $50 \mathrm{cmH}_{2} \mathrm{O} / \mathrm{mL} / \mathrm{seg} 7,15$.

Amamentação foi considerada quando os bebês foram alimentados no seio materno por um período mínimo de um mês.

\section{Análise estatística}

Foram calculadas as incidências de pneumonia, sibilos recorrentes e internações hospitalares por causa respiratória.

A associação entre as covariáveis sócio-demográficas, maternas, neonatais e pós-neonatais com a morbidade respiratória na idade entre 12 e 36 meses foi verificada pelo modelo linear generalizado com distribuição de Poisson e função de ligação logarítmica. A medida de associação produzida por este modelo linear generalizado é a razão de taxas de incidência de morbidade respiratória (número de episódios de morbidade/pessoa-tempo) entre crianças expostas e não expostas. Os resultados foram expressos como razões de taxas (RT) ajustadas com intervalo de 95\% de confiança (IC95\%). O teste da razão de verossimilhança foi usado para avaliar a associação entre cada covariável e a taxa de incidência de morbidade respiratória. Para minimizar o efeito do viés de memória devido ao longo período interconsultas e valorizar a informação dada pelas famílias de crianças que seguiram a rotina de comparecimento pré-estabelecida, todos os modelos foram ajustados pelo número total de consultas no período de estudo. Numa primeira etapa, foram ajustados modelos individuais para cada uma das covariáveis. Posteriormente, foram construídos diferentes modelos MLG ajustados por todas as covariáveis que apresentaram significância estatística no nível de $10 \%$ na análise prévia para avaliar o efeito de cada covariável neonatal/pós-neonatal.

Para a criação do banco de dados e análises descritivas preliminares, foi utilizado o programa Epi Info versão 3.2 (Centers for Disease Control and Prevention, Atlanta, Estados Unidos). Para a análise estatística, foi utilizado o programa $\mathrm{R}$ versão 2.7.0 (The R Foundation for Statistical Computing, Viena, Áustria; http://www.r-pro ject.org).

O presente estudo faz parte de um projeto que foi aprovado pelo Comitê de Ética em Pesquisa do IFF/FIOCRUZ, e os pais das crianças 
incluídas neste estudo assinaram o termo de consentimento informado.

\section{Resultados}

Foram admitidas na coorte 97 crianças. Dessas, uma foi a óbito aos três meses de vida por pneumonia e 12 não compareceram às consultas no ambulatório de seguimento no segundo ou terceiro anos de vida, permanecendo no presente estudo, portanto, um total de 84 crianças. Não houve diferença significativa entre as características neonatais do grupo de perdas e as dos participantes do estudo.

Durante o acompanhamento, cada criança fez em média três consultas (desvio-padrão - DP = 1,6) no segundo ano e duas consultas no terceiro ano ( $\mathrm{DP}=1,8)$. Foi elevada a freqüência das mães que apresentaram baixa escolaridade ( $57 \%$ com 8 anos ou menos de estudo) e baixa renda familiar $(79 \%$ com renda inferior ou igual a 2 salários mínimos). Segundo informação dos responsáveis, quase um quarto das mães fumou durante a gestação e $82 \%$ fizeram 3 ou mais consultas pré-natal.
Na Tabela 1, encontram-se algumas características da população estudada. A média da idade gestacional foi de 28 semanas ( $\mathrm{DP}=2,4)$, o peso médio ao nascimento foi de $1.097 \mathrm{~g}(\mathrm{DP}=236,2)$.

No período neonatal, houve utilização de surfactante em 28 recém-nascidos (33,3\%), e 8 $(9,5 \%)$ receberam corticoterapia. Pouco mais que $27 \%$ das crianças necessitaram de oxigenoterapia por 28 dias ou mais e cerca de $10 \%$ ainda faziam uso de oxigênio com 36 semanas de idade corrigida. Entre 12 e 24 meses, 45 das 80 crianças (56,3\%) apresentaram morbidade respiratória e procuraram assistência médica, enquanto, entre 24 e 36 meses, 24 das 63 crianças $(38,1 \%)$ avaliadas nesta faixa etária foram acometidas. Bronquiolite foi diagnosticada clinicamente em $23 \%$ das crianças. O tempo médio de amamentação no grupo de estudo foi de 4,7 meses (DP = 3,6).

No primeiro ano de vida, do total de 84 crianças, 46 (54,8\%) apresentaram morbidade respiratória, e 20 (23,8\%) crianças foram internadas, 30 $(35,7 \%)$ apresentaram pneumonia e $25(29,8 \%)$ apresentaram sibilos 7,8,9.

Ao comparar as incidências de morbidade respiratória nos períodos entre 12 a 24 meses e 24 e 36 meses, observa-se a redução de pneumo-

Tabela 1

Características da população de prematuros de muito baixo peso entre 12 e 36 meses de idade.

\begin{tabular}{|c|c|c|}
\hline Características da população estudada ( $N=84)$ & $\mathbf{n}$ & $\%$ \\
\hline \multicolumn{3}{|l|}{ Sexo } \\
\hline Masculino & 42 & 50,0 \\
\hline Feminino & 42 & 50,0 \\
\hline \multicolumn{3}{|l|}{ Renda domiciliar per capita (salários mínimos) } \\
\hline$<1$ & 36 & 42,9 \\
\hline $1-2$ & 31 & 36,9 \\
\hline$>2$ & 17 & 20,2 \\
\hline \multicolumn{3}{|l|}{ Escolaridade materna } \\
\hline 1ạ-8ạ série & 48 & 57,1 \\
\hline > 8a série & 36 & 42,9 \\
\hline Peso ao nascer inferior a $1.000 \mathrm{~g}$ & 27 & 32,1 \\
\hline Idade gestacional inferior a 28 semanas & 38 & 45,2 \\
\hline Uso de ventilação mecânica & 39 & 46,4 \\
\hline Doença de membrana hialina & 39 & 46,4 \\
\hline Persistência do canal arterial & 18 & 21,4 \\
\hline Pneumonia neonatal & 20 & 23,8 \\
\hline Displasia broncopulmonar & 23 & 27,4 \\
\hline Complacência pulmonar alterada & 36 & 42,8 \\
\hline Resistência pulmonar alterada & 53 & 63,1 \\
\hline Ter sido amamentado & 46 & 54,8 \\
\hline Tabagismo familiar & 31 & 36,9 \\
\hline Alergia familiar & 16 & 19,0 \\
\hline Morbidade respiratória no primeiro ano de vida & 46 & 54,8 \\
\hline
\end{tabular}


nia $(26,3 \%$ para $20,6 \%$ ) e sibilos ( $45 \%$ para $28,6 \%)$, porém, no que diz respeito à internação, houve um pequeno aumento de $8,8 \%$ para $9,5 \%$ no período entre 24 a 36 meses.

\section{Morbidade respiratória entre 12 e 36} meses e fatores associados

Os resultados da associação entre as co-variáveis e a morbidade respiratória ajustada somente pelo número de consultas (Tabela 2) mostram uma redução de $30 \%$ na RT de incidência de morbidade respiratória para as crianças que foram amamentadas (RT = 0,7; IC95\%: 0,5-0,9). Ter mais de uma criança no mesmo domicílio esteve associado com morbidade respiratória na idade estudada (RT = 1,5; IC95\%: 1,1-2,0). Sexo feminino, escolaridade materna superior a 8 anos, renda familiar entre 1 e 2 salários mínimos e idade gestacional inferior a 28 semanas também estiveram associados à taxa de incidência de morbidade respiratória. Esta análise preliminar também sugere que o uso de ventilação mecânica, displasia broncopulmonar, complacência pulmonar alterada, prematuridade extrema, pneumonia neonatal, persistência do canal arterial e morbidade respiratória no primeiro ano de vida estão associadas à morbidade respiratória.

A Tabela 3 apresenta os modelos multivariados para cada característica neonatal e pós-neonatal ajustados por todas as covariáveis com significância estatística na análise prévia (idade gestacional, sexo, escolaridade materna, renda familiar, ter mais de uma criança em casa). Tempo de amamentação, por ser considerada uma variável mediadora, não foi incluída nos modelos multivariados. A variável uso de ventilação mecânica no período neonatal apresentou significância estatística limítrofe (RT = 1,5; IC95\%: 1,02,1 ). Os diagnósticos de pneumonia, displasia broncopulmonar e persistência do canal arterial no período neonatal, complacência pulmonar diminuída na prova de função pulmonar e ter apresentado morbidade respiratória no primeiro ano de vida também se mantiveram associadas à morbidade respiratória na idade estudada.

Razão de taxas (RT) de incidência de morbidade respiratória em crianças entre 12 e 36 meses de idade nascidas prematuras de muito baixo peso.

\begin{tabular}{|c|c|c|}
\hline Covariáveis & RT ajustada * & IC95\% \\
\hline \multicolumn{3}{|l|}{ Sexo } \\
\hline Masculino & 1,00 & \\
\hline Feminino & 1,6 & $1,2-2,2$ \\
\hline \multicolumn{3}{|l|}{ Escolaridade } \\
\hline 1a-8ạ série & 1,00 & \\
\hline > 8ạ série & 1,5 & $1,1-2,1$ \\
\hline \multicolumn{3}{|l|}{ Renda domiciliar per capita (salários mínimos) } \\
\hline$<1$ & 1,00 & \\
\hline $1-2$ & 2,1 & $1,5-3,1$ \\
\hline$>2$ & 1,5 & $0,9-2,3$ \\
\hline Ter mais que uma criança em casa & 1,5 & $1,1-2,0$ \\
\hline Tabagismo na família & 0,9 & $0,6-1,3$ \\
\hline Alergia na família & 0,9 & $0,6-1,3$ \\
\hline Idade gestacional $<28$ semanas & 1,6 & $1,1-2,2$ \\
\hline Peso de nascimento $<1.000 \mathrm{~g}$ & 0,9 & $0,7-1,3$ \\
\hline Uso de ventilação mecânica & 1,5 & $1,1-2,0$ \\
\hline Displasia broncopulmonar & 2,1 & $1,5-2,9$ \\
\hline Complacência pulmonar alterada & 1,6 & $1,1-2,2$ \\
\hline Resistência pulmonar alterada & 0,9 & $0,6-1,3$ \\
\hline Pneumonia neonatal & 3,0 & $2,1-4,1$ \\
\hline Persistência do canal arterial & 1,9 & $1,4-2,7$ \\
\hline Ter sido amamentado & 0,7 & $0,5-0,9$ \\
\hline Morbidade respiratória no primeiro ano de vida & 2,1 & $1,5-3,0$ \\
\hline
\end{tabular}

* Ajustada pelo número de consultas. 
Razão de taxa (RT) de incidência de morbidade respiratória em prematuros de muito baixo peso entre 12 e 36 meses de idade segundo características neonatais e pós-neonatais.

\begin{tabular}{|c|c|c|}
\hline Covariáveis & RT ajustada * & IC95\% \\
\hline \multicolumn{3}{|l|}{ Características neonatais e pós-neonatais } \\
\hline Uso de ventilação mecânica & 1,5 & $1,0-2,1$ \\
\hline Displasia broncopulmonar & 1,9 & $1,2-2,9$ \\
\hline Complacência pulmonar alterada & 1,6 & $1,1-2,2$ \\
\hline Resistência pulmonar alterada & 0,9 & $0,7-1,4$ \\
\hline Pneumonia neonatal & 2,8 & $2,0-4,0$ \\
\hline Persistência do canal arterial & 1,6 & $1,1-2,4$ \\
\hline Morbidade respiratória no primeiro ano de vida & 1,8 & $1,3-2,6$ \\
\hline
\end{tabular}

* Ajustada por idade gestacional (contínua), sexo, nível educacional materno, renda familiar per capita, ter mais de uma criança em casa.

\section{Discussão}

\section{Morbidade respiratória e fatores associados}

Apesar de o sexo masculino ser freqüentemente mencionado na literatura como fator associado a maiores incidências de morbidade respiratória na infância, vale a pena ressaltar que no presente estudo o risco de morbidade respiratória para as meninas foi maior quando comparado ao dos meninos. Este paradoxo talvez seja explicado pelo fato das meninas terem apresentado doença neonatal mais grave, como maior incidência de displasia broncopulmonar $(31 \%$ e $23,8 \%$, respectivamente) e de persistência do canal arterial (23,8\% e $19 \%$, respectivamente). Da coorte original, um maior percentual de óbitos no período neonatal ocorreu em meninos. Além disso, mais crianças do sexo feminino nasceram com peso inferior a $1.000 \mathrm{~g}(33,3 \%$ meninas e $26,2 \%$ meninos). A incidência de morbidade respiratória no primeiro ano de vida também foi maior nas meninas do que nos meninos $(59,5 \%$ e $50 \%$, respectivamente). Nos modelos multivariados, nos quais os ajustes realizados tiveram por objetivo verificar o efeito das características neonatais e pós-neonatais na morbidade respiratória, foi observado que o efeito do sexo na presença das outras covariáveis se manteve contrário ao descrito na literatura.

Mulheres com escolaridade superior a 8 anos tendem a ter maior adesão aos serviços de saúde e maior valorização dos sintomas associados à morbidade respiratória. A população em estudo é constituída por um grupo relativamente homogêneo e de nível sócio-econômico desfavorável. A maior morbidade em relação a um nível mais elevado de renda e de escolaridade pode ser por conta de um viés de seleção ocasionado por uma menor sobrevivência dos casos mais graves nas faixas mais baixas de escolaridade e de renda, fazendo que os sobreviventes tivessem um menor risco de morbidade entre 12 e 36 meses de vida.

O tabagismo durante a gestação parece afetar o desenvolvimento pulmonar ocasionando menor diâmetro nas vias aéreas intrapulmonares 16, porém a associação entre tabagismo e morbidade respiratória não foi verificada (RT $=0,91$; IC95\%: 0,6-1,3), apesar de esta associação ser descrita na literatura 17.

No nosso estudo, as crianças que fizeram uso de ventilação mecânica no período neonatal apresentaram 1,5 vez o número de episódios de morbidade respiratória na idade entre 12 e 36 meses do que as não ventiladas, quando a razão de taxas foi ajustada somente pelo número de consultas. Entretanto, quando o modelo foi ajustado pela idade gestacional, sexo, escolaridade materna, renda familiar e ter mais de uma criança em casa, o uso de ventilação mecânica, apesar de apresentar magnitude de efeito inalterado, apresentou significância estatística limítrofe (RT = 1,5; IC95\%: 1,0-2,1). Resultados similares foram apresentados por Kinali et al. 18 e Thomas et al. 19 , que pesquisaram fatores preditivos para sibilância de repetição na infância. Thomas et al. ${ }^{19}$ constataram que o tempo do uso de ventilação mecânica, quando analisado junto com alterações radiográficas, perdeu a significância estatística.

A presença de pneumonia neonatal foi o fator de risco de maior magnitude da associação com a morbidade respiratória entre 12 e 36 meses, aumentando o risco 2,8 vezes (RT = 2,8; IC95\%: 2,0 $4,0)$. Isso confirma a hipótese de que o acometimento pulmonar mais grave no período neonatal 
está associado à morbidade respiratória na infância 20 em crianças que nasceram prematuras.

Rojas et al. ${ }^{21}$ relataram que o baixo peso ao nascer, persistência do canal arterial e sepse tiveram forte associação com doença pulmonar crônica no período neonatal em que as crianças que apresentaram persistência do canal arterial tiveram 6,2 vezes mais doença pulmonar crônica do que as crianças sem diagnóstico de persistência do canal arterial (IC95\%: 2,1-18,4).

Na coorte estudada, as crianças que apresentaram persistência do canal arterial, quando comparadas com aquelas sem persistência do canal arterial, aumentaram o risco de morbidade respiratória entre 12 e 36 meses em $60 \%$, demonstrando que a presença de persistência do canal arterial está associada à doença pulmonar mais grave no período neonatal e mais seqüelas pulmonares a longo prazo.

\section{Prova de função pulmonar alterada}

O nascimento prematuro pode estar associado com a interrupção do desenvolvimento pulmonar normal que pode resultar em anormalidades na mecânica pulmonar 22.

Giffin et al. 23 observaram que a resistência aérea elevada ( $\geq 50 \mathrm{cmH}_{2} \mathrm{O} / \mathrm{L} / \mathrm{s}$ ) com um ano de idade foi associada a maior risco de sintomas respiratórios como tosse e/ou sibilos no primeiro, segundo e terceiro anos de vida dos prematuros. Em oposição a esses resultados, a alteração da resistência pulmonar nas crianças acompanhadas na nossa coorte não teve associação com a morbidade respiratória (RT = 0,9; IC95\%: 0,7-1,4). Uma possível explicação é porque tais bebês nasceram após o advento do surfactante, portanto necessitaram de menos ventilação mecânica que os de outrora e também pelo fato de o maior comprometimento pulmonar ser intersticial e não de vias aéreas 7. Contudo, alteração na complacência pulmonar próximo à alta do berçário aumentou em $60 \%$ a taxa de incidência de morbidade respiratória entre 12 e 36 meses, quando comparadas às crianças que apresentaram complacência normal (IC95\%: 1,1-2,2). A metodologia empregada por Giffin et al. 23 foi diferente da por nós empregada, o que dificulta comparações.

\section{Sibilos recorrentes}

Os sibilos recorrentes foram a intercorrência respiratória mais incidente no grupo de crianças acompanhadas na nossa coorte. No período de 12 a 24 meses, 36 (45\%) das 80 crianças acompanhadas no ambulatório apresentaram sibilos e, no período de 24 a 36 meses, 18 (28,6\%) das 63 crianças foram acometidas.
Giffin et al. 23 observaram 88 crianças prematuras com peso médio ao nascer de $1.248 \mathrm{~g}$, acompanhadas até três anos de idade. As crianças foram consideradas sintomáticas quando apresentaram sibilos e/ou tosse por mais de três dias por semana por um período de quatro semanas ou se apresentassem sibilos e/ou tosse por pelo menos três dias após infecção do trato respiratório superior. Do total de crianças, 40 (45\%) foram sintomáticas no primeiro ano de vida, 30 crianças (34\%) no segundo ano e 28 crianças (32\%) no terceiro ano. É provável que as diferenças nas incidências comparadas às dos autores acima possam ser explicadas parcialmente pela utilização de diferentes critérios para a definição dos sintomas respiratórios.

No estudo de Elder et al. 11, 14,5\% dos que nasceram prematuros com menos de 33 semanas e $3 \%$ dos que nasceram a termo apresentaram sibilos recorrentes no primeiro ano de vida. Outros fatores associados à sibilância no grupo de crianças prematuras foram: presença de irmãos em casa (odds ratio - OR = 2,45; IC95\%: 1,39-4,31), hábito tabagista da mãe $(\mathrm{OR}=1,89$; IC95\%: 1,10$3,24)$, uso de oxigenoterapia por um período superior a 28 dias (OR = 3,09; IC95\%: 1,45-6,57) e com 36 semanas de idade corrigida $(\mathrm{OR}=4,25$; IC95\%: 1,93-9,35).

Pérez et al. 24 observaram, aos dois anos, a ocorrência de pelo menos um episódio de sibilo em $86,2 \%$ das crianças prematuras com história de displasia broncopulmonar, em $41,4 \%$ das crianças prematuras sem displasia broncopulmonar e em $18,8 \%$ do grupo a termo. No mesmo estudo, 17 das 29 crianças do grupo com displasia broncopulmonar continuaram sendo acompanhadas até a idade de quatro anos. A incidência de sibilos diminuiu de $88,2 \%$ no primeiro ano para $41 \%$ na idade entre 3 e 4 anos ( $<<0,001$ ).

Verificamos que não houve diferença significativa entre as incidências de sibilos recorrentes no segundo ano, enquanto no terceiro ano as crianças com displasia broncopulmonar foram 2,3 vezes mais acometidas do que as crianças sem displasia broncopulmonar ( $\mathrm{p}=0,03$ ) (dados não apresentados). Nossos achados sugerem que a prematuridade em si deva ter um papel importante na incidência de sibilos em crianças menores (12 a 24 meses), pois os dois grupos (com e sem displasia broncopulmonar) apresentaram incidências semelhantes de sibilos nesta faixa etária.

As crianças com idade gestacional inferior ou igual a 28 semanas tiveram risco 1,7 vez maior de apresentar sibilos recorrentes aos três anos, quando comparadas a crianças nascidas com idade gestacional maior do que 28 semanas 25 .

Encontramos resultados semelhantes aos do estudo realizado por Giffin et al. 25. As crian- 
ças com idade gestacional inferior a 28 semanas apresentaram número de episódios de morbidade respiratória entre 12 e 36 meses 1,6 vez maior do que as crianças com idade gestacional superior a 28 semanas (IC95\%: 1,1-2,2).

\section{Pneumonia}

No presente estudo constatou-se que ter mais de uma criança no domicílio esteve associado à maior incidência de morbidade respiratória na faixa etária estudada.

A aglomeração domiciliar está associada à doença infecciosa do trato respiratório inferior. Em um estudo caso-controle com crianças na faixa etária de 2 a 59 meses, Cardoso et al. 26 constataram um risco 2,5 vezes maior das crianças que vivem em aglomeração domiciliar apresentarem infecções respiratórias. Outro estudo constatou que onde dois ou mais moradores dividem o mesmo quarto há um aumento de $44 \%$ no risco de ter pneumonia $(\mathrm{OR}=1,44$; IC95\%: 1,06-1,96) 27.

No modelo estatístico adotado com ajuste apenas para o número de consultas, verificou-se que as crianças que residiam com outras crianças no mesmo domicílio apresentaram cerca de 1,5 mais episódios de morbidade respiratória na idade de 12 a 36 meses (IC95\%: 1,1-2,0). Estes dados são similares aos encontrados nos estudos supracitados 26,27 , provavelmente pelo fato de as condições sócio-econômicas das populações estudadas apresentarem características semelhantes às das crianças do nosso estudo.

No presente estudo, a incidência de pneumonia nas crianças entre 12 e 24 meses de idade foi de $26,3 \%$ e entre 24 e 36 meses, 20,6\%. Hack et al. 28 avaliaram 249 crianças com peso ao nascer inferior a $1.500 \mathrm{~g}$ e 363 controles nascidos a termo aos oito anos de idade. Os autores verificaram que das 249 crianças prematuras, $21(8,4 \%)$ tiveram diagnóstico de pneumonia após o período neonatal, enquanto somente 5 $(1,4 \%)$ das 363 crianças a termo foram acometidas por pneumonia neste mesmo período $(p<0,001)$. Estes resultados foram diferentes dos encontrados no nosso estudo. O estudo de Hack et al. 28 foi realizado em um país desenvolvido, onde há melhores condições sócio-econômicas e a população tem mais acesso aos serviços de saúde, e as crianças foram avaliadas numa idade mais tardia, além de ser diferente o critério utilizado para a coleta da informação. Ao passo que a população deste estudo vive em habitações precárias, com maior densidade domiciliar, geralmente com outras crianças menores de cinco anos, além de ter grande dificuldade de acesso à assistência médica, fato que aumenta o risco de adquirir uma doença infecciosa do trato respiratório e de a doença se agravar. Ainda como agravante, tais crianças não foram imunizadas contra pneumococo e não receberam profilaxia para vírus sincicial respiratório.

No estudo realizado por Victora et al. 27, crianças com história prévia de pneumonia ou hiper-reatividade brônquica tiveram chance até 2,5 vezes maior de apresentar pneumonia aos dois anos de idade (OR = 2,46; IC95\%: 1,36-4,43 e OR = 1,75; IC95\%: 1,16-2,63).

Autores relatam que o tipo de leite consumido pela criança também está associado à pneumonia. Crianças recebendo exclusivamente leite artificial no momento da entrevista tiveram risco três vezes maior de apresentar pneumonia do que as alimentadas com leite materno 27 . Observamos no presente estudo que as crianças alimentadas no seio materno apresentaram menos episódios de morbidade respiratória na idade entre 12 e 36 meses do que as que não foram amamentadas.

As crianças que apresentaram morbidade respiratória prévia (no primeiro ano de vida) tiveram 1,8 vez mais episódios de morbidade respiratória entre 12 e 36 meses. Esta associação também foi descrita por Prietsch et al. 29.

\section{Internações hospitalares}

Alguns estudos mostraram uma relação inversa entre a necessidade de internação hospitalar e a idade. Furman et al. 30 acompanhando prematuros de muito baixo peso ao nascer nos primeiros dois anos de vida, encontraram que 50\% deles necessitaram de ser internados no primeiro ano, enquanto $37 \%$ foram no segundo ano. No presente estudo, a necessidade de internação foi semelhante no segundo e terceiro anos de vida. Além disso, a incidência de hospitalizações foi bem menor (aproximadamente 10\%). Porém, esta mesma coorte no primeiro ano de vida apresentou um percentual de $23,8 \%$ de internação, revelando também uma redução do percentual de crianças internadas em relação aos dois anos posteriores. As crianças desta coorte nasceram após o advento do surfactante e da utilização de técnicas ventilatórias mais gentis. Tais fatores podem ter contribuído para o desenvolvimento de um quadro de menor gravidade pulmonar neonatal e menor incidência de internações em relação ao descrito nas crianças dos estudos aqui referidos, realizados na década de 1990. Outro aspecto a ser considerado é o fato de não estarem disponíveis informações sobre o desfecho de 12 crianças que não compareceram às consultas no período de 12 a 24 meses, apesar do empenho da equipe. Esta perda, fato freqüentemente relatado 
nos estudos de coorte, representa uma limitação do estudo.

\section{Conclusão}

As causas mais freqüentes de adoecimento de crianças menores de cinco anos e de demanda aos serviços de saúde são as afecções do aparelho respiratório 13. Apesar da distribuição global das doenças do aparelho respiratório, seu impacto sofre diferenças significativas de acordo com as desigualdades sócio-econômicas 27 . No presente estudo, foi abordada uma população particularmente vulnerável, os prematuros de muito baixo peso.

Pneumonia neonatal e displasia broncopulmonar foram associadas à maior incidência de morbidade respiratória na infância no período entre 12 e 36 meses, assim como a persistência do canal arterial e a presença de complacência pulmonar alterada no período anterior à alta da unidade neonatal. Fatores pós-neonatais como a presença de morbidade respiratória no primeiro ano de vida e fatores que refletem as condições de vida da nossa população - como o maior número de crianças no domicílio - aumentaram a taxa de morbidade respiratória nesta faixa etária.

$\mathrm{O}$ presente estudo mostra que a incidência de morbidade respiratória na idade entre $12 \mathrm{e}$ 36 meses se mantém elevada neste grupo de crianças de alto risco, especialmente entre 12 a 24 meses (56,3\%), o que reforça a necessidade de acompanhamento e de intervenções específicas efetivas na prevenção do adoecimento e na melhoria da qualidade de vida das crianças e suas famílias.

\section{Resumo}

O objetivo do estudo foi estimar a morbidade respiratória entre 12 e 36 meses em crianças prematuras e identificar os fatores associados. A população compreendeu 84 crianças de uma coorte de prematuros de muito baixo peso. O desfecho foi a taxa de incidência de morbidade respiratória. A associação entre as variáveis independentes e morbidade respiratória foi verificada por modelo linear generalizado. Entre 12 e 24 meses, 56,3\% das crianças apresentaram morbidade respiratória. Entre 24 e 36 meses, 38,1\% das crianças foram acometidas. As variáveis associadas à morbidade respiratória foram: displasia broncopulmonar ( $R T=1,9$; IC95\%: 1,2-2,9), complacência pulmonar alterada (RT = 1,6; IC95\%: 1,1-2,2), pneumonia neonatal ( $R T=2,8$; IC95\%: 2,0-4,0), persistência do canal arterial (RT = 1,6; IC95\%: 1,1-2,4) e morbidade respiratória no primeiro ano de vida ( $R T=1,8 ;$ IC95\%: 1,3-2,6). A incidência de morbidade respiratória entre 12 e 36 meses se manteve elevada neste grupo de crianças de alto risco, o que reforça a necessidade de acompanhamento e de intervenções efetivas na prevenção do adoecimento e na melhora da qualidade de vida destas crianças e suas famílias.

Prematuro; Recém-Nascido de Baixo Peso; Doenças Respiratórias

\section{Colaboradores}

G. Chalfun foi a responsável principal pela concepção geral do estudo, pela coleta e análise dos dados e redação do artigo. R. R. Mello participou da concepção geral do estudo, da coleta e análise dos dados e da redação do artigo. M. V. P. Dutra e K. S. Silva colaboraram na análise dos dados, redação do artigo e na sua análise crítica. V. L. Andreozzi participou da análise dos dados e de sua análise crítica. Todos os autores leram e aprovaram a versão final.

\section{Agradecimentos}

Este trabalho recebeu apoio da Fundação Oswaldo Cruz (FIOCRUZ) e Conselho Nacional de Desenvolvimento Científico e Tecnológico (CNPq) (PAPES IV). 


\section{Referências}

1. Greenough A, Giffin FJ, Yüksel B. Respiratory morbidity in preschool children born prematuraly. Relationship to adverse neonatal events. Acta Paediatr 1996; 85:772-7.

2. McCormick MC, Workman-Daniels K, BrooksGunn J, Peckham GJ. Hospitalization of very low birth weight children at school age. J Pediatr 1993; 122:360-5.

3. Gross SJ, Iannuzzi DM, Kveselis DA, Anbar RD. Effect of preterm birth on pulmonary function at school age: a prospective controlled study. J Pediatr 1998; 133:188-92.

4. Smith VC, Zupancic JAF, McCormick MC, Croen LA, Greene J, Escobar G, et al. Rehospitalization in the first year of life among infants with bronchopulmonary dysplasia. J Pediatr 2004; 144:799-803.

5. Anand D, Stevenson CJ, West CR, Pharoah POD. Lung function and respiratory health in adolescents of very low birth weight. Arch Dis Child 2003; 88:135-8.

6. Caetano JRM, Bordin IAS, Puccini RF, Peres CA. Fatores associados à internação hospitalar de crianças menores de cinco anos, São Paulo, SP. Rev Saúde Pública 2002; 36:285-91.

7. Mello RR, Dutra MV, Ramos JR, Daltro P, Boechat M, Andrade-Lopes JM. Lung mechanics and highresolution computed tomography of the chest in very low birth weight premature infants. São Paulo Med J 2003; 121:167-72.

8. Mello RR, Dutra MV, Lopes JMA. Morbidade respiratória no primeiro ano de vida de prematuros egressos de uma unidade pública de tratamento intensivo. J Pediatr (Rio J) 2004; 80:503-10.

9. Mello RR, Dutra MVP, Ramos JRM, Boechat MC, Daltro P, Lopes JMA. Neonatal risk factors for respiratory morbidity in the first year of life among premature infants. São Paulo Med J 2006; 124:77-84.

10. Jobe AH, Bancalari E. Bronchopulmonary dysplasia. Am J Respir Crit Care Med 2001; 163:1723-9.

11. Elder DE, Hogan R, Evans SF, Benninger HR, French NP. Recurrent wheezing in very preterm infants. Arch Dis Fetal Neonatal Ed 1996; 74:165-71.

12. Gibson GJ. Pulmonary hyperinflation - a clinical overview. Eur Respir J 1996; 9:2640-9.

13. Cesar JA, Victora CG, Santos IS, Barros FC, Albernaz EP, Oliveira LM, et al. Hospitalização por pneumonia: influência de fatores socioeconômicos e gestacionais em uma coorte de crianças no sul do Brasil. Rev Saúde Pública 1997; 31:53-61.

14. Shennan AT, Dunn MS, Ohlsson A, Lennox K, Hoskins EM. Abnormal pulmonary outcomes in premature infants: prediction of oxygen requirements in the neonatal period. Pediatrics 1988; 82:527-32.

15. Abasi S, Bhutani V. Pulmonary mechanics and energetics of normal, non-ventilated low birthweight infants. Pediatr Pulmonol 1990; 8:89-95

16. Martinez FD. Definition of pediatric asthma and associated risk factors. Pediatr Pulmonol Suppl 1997; 15:9-12.
17. Gonçalves-Silva RMV, Valente JG, Lemos-Santos MGF, Sichieri R. Tabagismo no domicílio e doença respiratória em crianças menores de cinco anos. Cad Saúde Pública 2006; 22:579-86.

18. Kinali M, Greenough A, Dimitriou G, Yuksel B, Hooper R. Chronic respiratory morbidity following premature delivery- prediction by prolonged respiratory support requirement? Eur J Pediatr 1999; 158:493-6.

19. Thomas M, Greenough A, Johnson A, Limb E, Marlow N, Peacock JL, et al. Frequent wheeze at follow up of very preterm infants: which factors are predictive? Arch Dis Child 2003; 88:329-32.

20. Bancalari E, Gerhardt T. Bronchopulmonary dysplasia. Pediatr Clin North Am 1986; 33:1-23.

21. Rojas MA, Gonzales A, Bancalari E, Claure N, Poole C, Silva-Neto G. Changing trends in the epidemiology and pathogenesis of neonatal chronic lung disease. J Pediatr 1995; 126:605-10.

22. Greenough A, Dimitriou G, Bhat RY, Broughton S, Hannam S, Rafferty GF. Lung volumes in infants who had mild to moderate bronchopulmonary dysplasia. Eur J Pediatr 2005; 164:583-6.

23. Giffin F, Greenough A, Yüksel B. Relationship between lung function results in the first year of life and respiratory morbidity in early childhood in patients born prematurely. Pediatr Pulmonol 1994; 18:290-4.

24. Pérez GP, Merino MN, Pérez MMR, Reguera CS, Tubio AP, Padillo JP. Morbilidad respiratoria tras el alta hositalaria en prematuros ( $<32$ semanas) con displasia broncopulmonar. An Pediatr (Barc) 2004; 60:117-24

25. Giffin F, Greenough A, Yüksel B. Prediction of respiratory morbidity in the third year of life in children born prematurely. Acta Paediatr 1994; 83:157-8.

26. Cardoso MRA, Cousens SN, Siqueira LFG, Alves FM, D'Angelo LA. Crowding: risk factor or protective factor for lower respiraratory disease in young children? BMC Public Health 2004; 4:19.

27. Victora CG, Fuchs SC, Flores JAC, Kirkwood B. Risk factors for pneumonia among children in a brazilian metropolitan area. Pediatrics 1994; 93(6 Pt 1): 977-85.

28. Hack M, Weissman B, Breslau N, Klein N, Borawski-Clark E, Fanaroff AA. Health of very low birth weight children during their first eight years. J Pediatr 1993; 122:887-92.

29. Prietsch SOM, Fischer GB, César JA, Lempek BS, Barbosa Jr. LV, Zogbi L, et al. Acute lower respiratory illness in under-five children in Rio Grande, Rio Grande do Sul State, Brazil: prevalence and risk factors. Cad Saúde Pública 2008; 24:1429-38.

30. Furman L, Baley J, Borawski-Clark E, Aucott S, Hack M. Hospitalization as a mesure of morbidity among very low birth weight infants with chronic lung disease. J Pediatr 1996; 128:447-52.

Recebido em 22/Ago/2008

Versão final reapresentada em 15/Jan/2009

Aprovado em 13/Fev/2009 